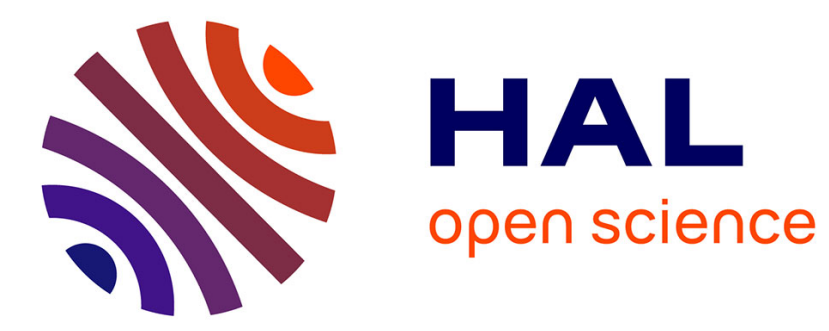

\title{
Structure and function of polycystins: insights into polycystic kidney disease
}

Dominique Douguet, Amanda Patel, Eric Honore

\section{To cite this version:}

Dominique Douguet, Amanda Patel, Eric Honore. Structure and function of polycystins: insights into polycystic kidney disease. Nature Reviews Nephrology, 2019. hal-02378835

\section{HAL Id: hal-02378835 \\ https://hal.science/hal-02378835}

Submitted on 27 Nov 2019

HAL is a multi-disciplinary open access archive for the deposit and dissemination of scientific research documents, whether they are published or not. The documents may come from teaching and research institutions in France or abroad, or from public or private research centers.
L'archive ouverte pluridisciplinaire HAL, est destinée au dépôt et à la diffusion de documents scientifiques de niveau recherche, publiés ou non, émanant des établissements d'enseignement et de recherche français ou étrangers, des laboratoires publics ou privés. 


\title{
Structure and function of polycystins: insights into polycystic kidney disease
}

\author{
Dominique Douguet ${ }^{1}$, Amanda Patel ${ }^{1}$ and Eric Honoré ${ }^{1 *}$ \\ ${ }^{1}$ Université Côte d'Azur, Centre National de la Recherche Scientifique, Institut national de la santé et \\ de la recherche médicale, Institut de Pharmacologie Moléculaire et Cellulaire, Labex ICST, Valbonne, \\ France. \\ *Email: honore@ipmc.cnrs.fr
}

\begin{abstract}
Mutations in the polycystins PC1 or PC2 cause autosomal dominant polycystic kidney disease (ADPKD), which is characterized by the formation of fluid-filled renal cysts that disrupt renal architecture and function, ultimately leading to kidney failure in the majority of patients. Although the genetic basis of ADPKD is now well established, the physiological function of polycystins remains obscure and a matter of intense debate. The structural determination of both the homomeric PC2 and heteromeric PC1-PC2 complexes, as well as the electrophysiological characterization of PC2 in the primary cilium of renal epithelial cells, provided new valuable insights into the mechanisms of ADPKD pathogenesis. Current findings indicate that PC2 can function independently of PC1 in the primary cilium of renal collecting duct epithelial cells to form a channel that is mainly permeant to monovalent cations and is activated by both membrane depolarization and an increase in intraciliary calcium. In addition, PC2 functions as a calcium-activated calcium release channel at the endoplasmic reticulum membrane. Structural studies indicate that the heteromeric PC1-PC2 complex comprises one PC1 and three PC2 channel subunits. Surprisingly, several positively-charged residues from PC1 occlude the ionic pore of the PC1-PC2 complex, suggesting that pathogenic polycystin mutations might cause ADPKD independently of an effect on channel permeation. Emerging reports of novel structural and functional findings on polycystins will continue to elucidate the molecular basis of ADPKD.
\end{abstract}

\section{Main text}

Autosomal dominant polycystic kidney disease (ADPKD) is one of the most common human monogenic diseases, with a prevalence of about 1 in $1000^{1,2}$. This multi-system inherited disorder is characterized by the progressive development of fluid-filled cysts in the kidney, liver and pancreas, and is associated with hypertension, kidney failure and brain aneurysms ${ }^{3,4}$. Mutations that cause ADPKD occur on PKD1 and PKD2, which encode polycystin 1 (PC1; also known as PKD1) and polycystin 2 (PC2; also known as PKD2 or TRPP2), respectively ${ }^{5,6}$. Mutations in PKHD1, which encodes fibrocystin (also known as polyductin), cause the autosomal recessive form of the disease (ARPKD) ${ }^{7}$. One study proposed that the carboxy-terminal domain of fibrocystin binds to the intracellular amino terminus of PC2 and that loss of fibrocystin results in reduced PC2 expression ; however, another study did not confirm this interaction between fibrocystin and PC2 ${ }^{8,9}$. Additional reported partners for PC2 include other ion channel subunits such as the transient receptor potential (TRP) channels TRPV4 and TRPC1, and the Piezo1 mechanosensitive ion channel ${ }^{10-15}$. 
Genetically, ADPKD is autosomal dominant as the mutations associated with the disease are heterozygous. However, at the cellular level, cyst formation requires a second somatic mutation in the normal allele that results in either reduced expression or inactivation of the wild-type gene ${ }^{16-18}$; these pathogenic mutations affect intracellular calcium homeostasis, as well as numerous additional signaling pathways ${ }^{19}$. Notably, in ADPKD, low cytosolic calcium concentration is associated with enhanced CAMP levels, due to calcium-dependent regulation of adenylate cyclase and phosphodiesterases, which are responsible for the synthesis and degradation of cAMP, respectively. The rise in intracellular CAMP promotes the proliferation of tubular epithelial cell and cystic fibrosis transmembrane conductance regulator (CFTR)-mediated fluid secretion ${ }^{20-22}$.

The genetic basis of ADPKD is well known, including the identity of the mutated genes and mutational mechanisms, but the biological function of polycystins remains poorly understood. Initial reports indicated that PC1 and PC2 combine to form a calcium-permeable channel at the plasma membrane ${ }^{23,24}$. However, subsequent studies in which PC1 expression was targeted with small interfering RNAs (siRNAs) or through a conditional knockout of PKD1 challenged this assertion ${ }^{25,26}$. Instead, those data suggested that PC2 can function independently of PC1 as a cationic channel with low calcium permeability; this function seems to be restricted to the primary cilium [G] and is not observed in other areas of the plasma membrane. Moreover, some reports indicated that PC1-PC2 complexes in the primary cilium of both renal tubular epithelial cells and endothelial cells are responsible for sensing shear stress ${ }^{27-29}$, whereas subsequent findings showed that primary cilia are not calcium-responsive mechanosensors ${ }^{30}$.

The resolution of the atomic structure of both PC1 and PC2 addressed a major gap in the field and provided valuable new insights into the permeation [G] and gating mechanism [G] of the PC2 cationic channel; surprisingly, it also revealed a pore blocking function for PC1 ${ }^{31-35}$. In this Review, we describe the most recent structural and functional findings about polycystins and tentatively reconcile some of the earlier controversies in the field.

\section{[H1] Pre-structural studies of polycystins}

Mutations in PKD1 or PKD2 produce a similar cystic phenotype, suggesting that both proteins might function in the same pathway and/or complex ${ }^{1,36,37}$. Indeed, PC1 interacts with PC2 through coiled-coil domains [G] within the cytosolic carboxy-terminal domains ${ }^{38}$. It was initially reported that co-assembly of PC1 and PC2 resulted in a non-selective [G] cation-permeable current at the plasma membrane when overexpressed in transfected Chinese hamster ovary $(\mathrm{CHO})$ cells, whereas neither PC1 nor PC2 alone could produce a current ${ }^{23}$. In the absence of PC1, PC2 was retained in the endoplasmic reticulum (ER) and only translocated to the plasma membrane when co-expressed with 
PC1 ${ }^{23}$; PC2 contains a C-terminal ER retention motif ${ }^{39}$. One of the disease-causing mutated forms of $P K D 1, \mathrm{R} 4227 \mathrm{X}$, encodes a truncated PC1 protein that lacks the last 76 amino acids, which include the coiled-coil domain. Notably, co-expression of this mutated isoform of PC1, which cannot interact with PC2, did not result in a measurable current ${ }^{23}$. Similarly, co-expression of full-length PC1 with PC2 742X, a pathogenic mutated isoform of PC2 that lacks the last 227 amino acids, including the coiled-coil domain, did not yield a measurable current ${ }^{23}$. PC1 was thus proposed to interact with PC2 and enable its trafficking to the plasma membrane where it established a non-selective cationpermeable current ${ }^{23}$. These findings were elegantly confirmed in rat sympathetic neurons, in which exogenous co-assembly of PC1 with PC2 was shown to form a functional ion channel complex at the plasma membrane ${ }^{24}$. This study also suggested that PC2 activation might result from a conformational change in PC1, as induced by an activating antibody raised against the extracellular domains of $\mathrm{PC} 1^{24}$.

These seminal reports put forward the notion that PC1 controls both the trafficking and the gating of PC2, and that pathogenic mutations in either subunit impair calcium-dependent signalling in renal tubular epithelial cells, as well as in other cell types affected by ADPKD, which include arterial smooth muscle cells ${ }^{23,24,40}$. Consequently, prior to the structural determination of polycystins, the dogmatic view was that PC1 acts as an obligatory positive regulator of PC2 ${ }^{23,24}$. Surprisingly, structural studies suggest an intriguing alternative hypothesis in which PC1 blocks cation permeation through the heteromeric PC1-PC2 complex ${ }^{33}$.

\section{[H1] Atomic structure of homomeric PC2}

$P C 2$, which is found at the membrane of the primary cilium and at the ER, belongs to the TRP ion channel family ${ }^{41}$ (FIG. 1b); its structure was first solved by cryogenic-electron microscopy (cryoEM) at a resolution of $3 \AA \AA$ in lipid nanodiscs [G] (BOX 1) ${ }^{31,32,34}$. PC2 forms a homotetramer and each subunit contains a pore domain, composed of two pore helices ( $\mathrm{PH} 1$ and $\mathrm{PH} 2$ ) and a selectivity filter [G], as well as a voltage sensor [G] -like domain (VSD) that is formed by S1-S4 (FIG.1b and 1d).

\section{[H2] The selectivity filter}

Alignment of the PC2 protein sequence with that of other TRP channel subunits suggests the presence of a selectivity filter between S5 and S6 (FIG. 1b) ${ }^{31,32,34}$. The external part of the pore is enriched in negative charges and probably acts as a cation sink [G] ${ }^{31}$; in addition, the pore is constricted within the selectivity filter between amino acids Leu641-Gly642-Asp643 (FIGs. 1d and 2), which narrows the ionic pathway so that only dehydrated cations can move through the pore ${ }^{31}$. 
Within the homotetramer, the negatively-charged Asp643 residues point to the center of the pore, presumably interacting with cations, while repulsing anions (FIG. 1e) ${ }^{31}$. The Leu-Gly-Asp sequence found in the PC2 selectivity filter is also present in PC2-like 1 protein, a PC2 homologue (71\% sequence similarity) encoded by PKD2L1 that is responsible for sour taste sensing and other $\mathrm{pH}$ dependent processes that occur at the primary cilium ${ }^{42,43}$. Since, unlike PC2, transfected PC2-like 1 protein is active at the plasma membrane, chimeric constructs that combined the pore domain of PC2 (Glu631-Pro658) with the core domain of PC2-like 1 protein have been used to evaluate the ionic selectivity of PC2 ${ }^{31}$. The data obtained from these constructs indicated that PC2 is mainly a sodium and potassium conducting channel (Px:PNa permeability ratio $\left.=2.2 \mathrm{~K}^{+} ; 1 \mathrm{Na}^{+} ; 0.5 \mathrm{Ca}^{2+}\right)^{31}$.

\section{[H2] Calcium binding sites}

The structural analysis of full length PC2 in complex with calcium and lipids revealed two distinct channel states ${ }^{34}$. The structure with a larger pore radius of $1.7 \AA$ presented a single cation located below the selectivity filter between Leu641 and Phe669, whereas the alternative structure with a smaller pore radius of $1.4 \AA$ (compared to the close state with a pore radius of $1.0 \AA$ ) contained five cation binding sites along the conduction pathway [G] (presumably a calcium-blocked state), interacting with Asp643 in the selectivity filter, three other sites between Leu641 and Phe669 and finally a fifth binding site at the channel exit, involving Asn681 and Ser685 ${ }^{34}$. These structural data support electrophysiological studies indicating that PC2 permeation to monovalent cations is blocked by extracellular calcium, presumably through binding of calcium to Asp643 within the selectivity filter ${ }^{25,26,34}$.

The cytosolic carboxy-terminal EF hand motif (FIG. 1b) [G], upsteam of the coiled coil domain, constitute a key regulatory domain that controls the activation of PC2 by intracellular calcium 25,26,34,39,44-46. The high-resolution structure of the EH-hand domain of human PC2 was determined using NMR spectroscopy ${ }^{47-49}$. The PC2 region $717-792$ was shown to bind with a low affinity $(122 \mu \mathrm{M})$ a single calcium ion and this binding was abolished when glutamate 774 was mutated in glutamine ${ }^{47}$. Therefore, whereas calcium binding to the cytosolic C-terminal EF hand motif results in the activation of PC2, extracellular calcium binding to Asp643 blocks permeation of monovalent cations ${ }^{25,26,31,39,49}$.

\section{[H2] The polycystin domain}

PC2 contains a large extracellular domain ( 200 amino acids long), termed the polycystin domain (PCD), which is also present in PC1 and PC2-like 1 protein (FIGs. $1 \mathrm{~b}$ and 1d). The PCD, found between S1 and S2 and extending $35 \AA$ above the membrane, is involved in the assembly of the ion channel subunits and might have a role in the regulation of channel gating ${ }^{31-34}$ (FIGs. $1 \mathrm{~b}, 1 \mathrm{~d}$ and $3 \mathrm{~b}$ ). 
The importance of PCDs is highlighted by the clinical data demonstrating that this region is a hot spot for pathogenic mutations (FIGs. 1d) ${ }^{31-34}$; moreover, PCD glycosylation is important for both the stability and trafficking of PC2 ${ }^{50}$. The PCD interacts with the pore of the adjacent subunit and the VSD of its own subunit ${ }^{31-34}$. Below the PCD and on top the selectivity filter, a large upper vestibule of highly negatively-charged amino acids is accessible from the extracellular side through an apical opening and four lateral portals ${ }^{34}$; lipids also bind to this interfacial region. One possibility is that the selectivity filter is blocked when the amphipathic [G] funnels below the PCD are filled with water ${ }^{34}$ (FIG. 3a). By contrast, interactions between lipids in the VSD and the PCD might enhance the permeation and/or activation of the channel by preventing water from accumulating in the lateral funnel, thus allowing the conduction of dehydrated cations through the selectivity filter ${ }^{34}$ (FIG. 3b). This model suggests that changes in the conformation of the PCD might contribute to channel activation ${ }^{34}$, in which case, the PCD might act as a lid on top of the channel and regulate channel activation in response to physical and/or chemical stimuli ${ }^{31}$ (FIG. 3b).

\section{[H2] The voltage sensing domain}

The S4 of the PC2 VSD only contains 2 of the 4 positive charges that are usually found in voltage-gated channels (FIG. 3c). Of note, these positive charges are facing Asp511 in S3, which is a frequent site of missense pathogenic mutations in patients with ADPKD. An alternative model to the aforementioned model of PCD-regulated activation of PC2 ${ }^{34}$ is that PC2 gating is controlled by conformational changes within the VSD (that is, segments S1-S4) ${ }^{43}$ (FIG. 3c). In this model, the PCD would remain mostly static upon membrane depolarization, whereas the positively charged voltage sensing domain $\mathrm{S} 4$ would be predicted to undergo a clockwise twist of about $4^{\circ}$ together with an outward movement of about $4 \AA$ (FIG. 3c). Such a conformational change in the VSD is anticipated to result in an iris-like opening of the lower end of S6, where the hydrophobic gate [G] (Leu677) that keeps the ionic pore closed is located ${ }^{43}$ (FIGs. 3a and 3c). The last nine residues of S4 (Ile571-Phe579) form a $3_{10}$ helical region (that is, 3 residues per turn) that might be involved in gating movements of the S4-S5 linker, which is coupled to S6 via a hydrogen bond between GIn585 and Lys688 ${ }^{31}$.

\section{[H2] The inner activation gate}

Before the publication of the structural studies, very limited information was available about the gating mechanisms of PC2 ${ }^{36,51}$. Leu677 at the cytoplasmic end of S6 forms a single residue hydrophobic barrier that is predicted to prevent ion permeation by repulsing water molecules at the cytoplasmic side of the channel ${ }^{35}$ (FIG. 2a). Accordingly, the non-pathogenic Leu677Asn or Leu677Gly mutants of PC2, which were created by systematic site-directed mutagenesis replacing Leu677 with polar residues, greatly enhanced PC2 currents ${ }^{35}$. Similarly, the Phe604Pro mutation, 
another experimental mutation that is not reported in patients, results in constitutive activation of PC2, probably by bending S5 and pulling on the Leu677 hydrophobic gate ${ }^{35,52}$ (FIGs. 2b and 3d). Similarly, the double experimental mutant Phe604Pro Leu677Gly caused very large PC2 currents ${ }^{35}$. The S6 of PC2 is characterized by the presence of a $\pi$ helix [G] at amino acids Met668-Phe-Phe-IleLeu672, which possibly acts as a point of flexibility that causes the rotation of the Leu677 hydrophobic gate so that it points away from the centre of the pore. In the Phe604Pro mutant, the inner pore diameter increases from $5 \AA$ to $7.6 \AA$, allowing cation permeation ${ }^{35}$ (FIGs. $2 b$ and 3d). No major difference was found in the diameter of the selectivity filter between the closed channel and the constitutively open channel composed of Phe604Pro mutant subunits, indicating that the inner hydrophobic gate Leu677 alone might control the opening of PC2 ${ }^{35}$ (FIG. 2). However, the selectivity filter might still possibly act as a secondary gate ${ }^{31}$. Interestingly, the gain-of-function mutants Phe604Pro and Leu677Gly rescued the cystic phenotypes induced by PKD2 knockdown in zebrafish more efficiently than wild-type PC2; this rescue effect was even more pronounced in the double mutant (Phe604Pro and Leu677Gly) ${ }^{35}$. These in vivo findings provide further functional evidence that both Phe604Pro and Leu677Gly are gain-of-function mutations that open the PC2 channel.

The structural studies of PC2 provide novel insights into the permeation, as well as the gating mechanisms of the PC2 ion channel. Currently no structure for PC1 alone is available and whether PC1 might form a biologically relevant multimer in the absence of PC2 is unknown. However, the reported structure of the PC1-PC2 heteromeric complex suggests an unexpected function for PC1 ${ }^{33}$.

\section{[H1] The heteromeric PC1-PC2 complex}

The structure of the PC1-PC2 complex was solved by cryo-EM at a $3.6 \AA$ resolution ${ }^{33}$ (BOX 1). The N-terminal domain of PC1 (NTD), formed by TM1-TM5, lies on the side of the PC1 VSD and contains a PC1 lipoxygenase and $\alpha$ toxin (PLAT) domain that is located between TM1 and TM2 and faces the cytosol ${ }^{33}$ (FIGs. 1a and 1c). The PLAT domain can be found in a variety of membraneassociated or lipid-associated proteins, where it is involved in lipid binding and/or trafficking. Of note, the PC1 protein used for the structural studies of PC1-PC2 lacked the large cleavable extracellular $\mathrm{N}$-terminal domain ${ }^{53}$.

The carboxy-terminal domain of the PC1 subunit is similar to that of PC2 and includes a VSD formed by S1 to S4, as well as a PCD between S1 and S2 (FIG. 1a-b). Importantly, sequences corresponding to the selectivity filter (SF) and the supporting pore helices (PH1 and PH2), as found in PC2, are absent in PC1 (FIG. 1c-d). However, a 29 amino acid long segment (4051-4080), within the predicted pore region, was not resolved in the PC1 structure ${ }^{53}$. The heteromeric PC1-PC2 complex is composed of one PC1 and three PC2 subunits, as previously suggested by studies of single molecules 
${ }^{54}$ (FIG. 1e). Early studies indicated that PC1 and PC2 interact through their carboxy-terminal coiledcoil domains ${ }^{38}$. However, the PC1 construct used for the subsequent structural determination of the complex showed that heteromultimerization with PC2 was possible, even in the absence of the cytosolic coiled-coil domains ${ }^{33}$. Similarly to the homotetrameric PC2 channel, the channel core of the PC1-PC2 heteromeric complex is formed by 24 transmembrane segments, but it also contains an additional 5 transmembrane segments from the amino-terminal domain of PC1, which renders the complex asymmetric (FIG. 1e). The four VSDs in the heteromeric complex show a pseudo four-fold symmetry, although the S6 of PC1 is bent in the middle of the helix (FIG. 1a and 1e). Strikingly, three positively-charged residues from the S6 - Arg4100, Arg4107 and His4111 -face the conduction pathway and are predicted to prevent cation permeation through the pore (FIG. 1e). These findings suggest that the heteromeric PC1-PC2 complex may be non-conductive and that PC1 could act as a dominant-negative subunit of the PC2 channel $^{33}$.

Modeling PC1 in an "activated" state (based on the Phe604Pro PC2 structure), shows that at least one positively-charged residue remains in the conduction pathway (D.D., unpublished observations), suggesting that permeation through the complex might be impaired, independently of PC1 activation. It will be interesting to study the co-expression of PC1 with PC2 Phe604Pro and/or Leu677Asn (constitutively open mutants forms of PC2) to functionally determine whether PC1 still inhibits opening of the activated PC2 channel.

These findings contrast with previous functional data, which suggested that PC1 is required for the channel activity of PC2 at the plasma membrane ${ }^{23,24}$. However, it remains possible that either direct or indirect binding of a specific ligand to the PC1-PC2 complex alters the conformation of PC1 and opens the channel ${ }^{24,55,56}$. Alternatively, auxiliary subunits that can complex with PC1 or PC2, such as other TRP channel subunits, might modify the structural properties of the complex and allow ion permeation through the heteromeric PC1-PC2 channel. Another possibility is that the absence of the coiled-coil domain in the construct used for structural determination might have favoured a heteromeric assembly between truncated PC1 and the PC2 subunits that is not physiologically relevant.

These structural studies support the notion that PC1 might prevent cation permeation through the PC1-PC2 ion channel complex (FIG. 1e), which is rather unexpected as both PKD1 and PKD2 loss-of-function mutations cause ADPKD. The only difference is that the phenotype associated with PKD2 mutations is milder than that associated with PKD1 mutations and is characterized by a lower number of cysts in renal parenchyma, although the rates of cyst growth are identical ${ }^{57}$. The difference in phenotype might be due to the difference in the size of both genes; the larger size of PKD1 might increase its probability of acquiring the required second hit mutation on the somatic allele ${ }^{57}$. 
In addition to ion permeation, the PC1-PC2 complex is also involved in regulation of the cell cycle, cell adhesion and intracellular signaling; disruption of any of these functions due to loss-offunction mutations in PKD1 or PKD2 could potentially contribute to polycystic disease. Notably, and similarly to PC2, the PCD of PC1 ${ }^{33}$ (FIG. 1a-b) is a hot spot for pathogenic mutations ${ }^{33}$ (FIG. 1c); the PC1 PLAT domain is another common site for pathogenic mutations ${ }^{33}$ (FIG. 1C). Strikingly, pathogenic mutations in the pore domain of PC1 have not been described, further suggesting that the pathogenic mechanisms of polycystin mutations might be independent of channel permeation (FIG. 1c). Clearly, further investigation will be needed to confirm and elucidate the significance of the reported structural findings.

\section{[H1] Ion channel functions of polycystins}

Polycystins are located at the membrane of the primary cilium and of the ER. The ion channel function of PC2 was demonstrated at both locations, activated by an increase in cytosolic calcium $25,26,39$

\section{[H2] Cationic channels at the primary cilium}

Single channel currents at the primary cilium of renal epithelial cells were first described in $2005^{58}$ and electrophysiological findings reported in 2017 and 2018 suggest that these currents are PC2-dependent ${ }^{25,26}$. The electrophysiological activity of native ciliary PC2 was recorded in a murine cell line derived from inner medullary tubules, mIMCD-3, as well as in primary cultures of mouse collecting duct cells (FIG. 4a-c); of note, cysts in ADPKD are mainly found in the renal medulla. The researchers recorded a large conductance non-selective cationic channel of $97 \mathrm{pS}$ in physiological solution that had a $\mathrm{PK}^{+}: \mathrm{PNa}^{+}$permeability ratio of 2.4 , indicating that potassium is more permeant than sodium (FIG. 4b-c). Importantly, knock out of PKD2 with CRISPR-Cas9 technology suppressed this cationic current in mIMCD-3 cells ${ }^{26}$; transfection of collecting duct cells with siRNAs against PKD2 or genetic deletion of this gene also eliminated cationic currents at the primary cilium ${ }^{25}$. Provocatively, PC2 channel activity at the primary cilium was still present in cells that lacked PC1 ${ }^{25}$, a finding that is again in sharp contrast with previous observations that suggest an obligatory role for PC1 in the regulation of PC2 trafficking to the plasma membrane and channel gating ${ }^{23,24}$. In line with this finding, the $\mathrm{N}$-terminal domain of $\mathrm{PC2}$ contains a ciliary trafficking motif $(\mathrm{R} 6 \mathrm{~V} \times \mathrm{P})^{59}$. However, the possibility that the conditional Pkd1 mice used to test the activity of PC2 in the absence of PC1 might have some residual PC1 expression in tubular cells cannot be disregarded ${ }^{25}$. In addition, these findings do not support an inhibitory function of PC1 on PC2 permeation, as suggested by the structural data ${ }^{33}$. One possibility for this discrepancy is that the trafficking of heteromeric PC1-PC2 
and homomeric PC2 complexes to the primary cilium occurs through independent pathways - in this scenario, inactivation of PKD1 might not influence the electrophysiological function of homotetrameric $\mathrm{PC2}$ at the primary cilium ${ }^{25}$. However, even if the basal activity of PC2, which is presumably driven by the homotetrameric PC2 complex, occurs independently of PC1, it is still possible that PC1 regulates the PC2 channel. For example, PC1 activation in response to the binding of a ligand such as Wnt might result in the activation the PC1-PC2 heteromeric complex ${ }^{55}$. Binding of Wnt to the extracellular domain of PC1 has been shown to result in PC2-dependent calcium influx ${ }^{55}$, although subsequent findings from experiments that used HEK and $\mathrm{CHO}$ cells co-transfected with both PC1 and PC2 have challenged the potential role of Wnt in PC2 activation ${ }^{31}$. Further experiments will be required to explain whether or not additional subunits and/or receptors are involved in the possible regulation of PC1-PC2 by Wnt.

\section{[H3] Calcium permeation}

The selectivity of PC2 for calcium is controversial as one group reported a signficant $\mathrm{PCa}^{2+}: \mathrm{PK}^{+}$ permeability ratio of $0.55^{26}$, whereas another study reported a negligible $\mathrm{PCa}^{2+}$ : $\mathrm{PK}^{+}$ratio of 0.025 , which suggested instead that PC2 is poorly permeant to calcium ${ }^{25,26}$. However, such low calcium permeability through PC2 ${ }^{25}$ is at odds with previous findings from functional studies $23,24,27,29,39,40$. Moreover, ion channel chimeras in which the selectivity filter of PC2 was inserted into the core of PC2-like 1 protein, also resulted in substantial calcium permeability $\left(\mathrm{PCa}^{2+}: \mathrm{PNa}^{+}\right.$permeability ratio of $0.5)^{31}$. However, both the pore dilation and electrostatic fields of PC2-like protein 1 might influence calcium permeability of the chimeric channel ${ }^{25,42}$. Whether native PC2 permeates calcium therefore remains an open question.

\section{[H3] Activation of PC2 at the primary cilium}

The latest electrophysiological findings indicate that PC2 functions as a cationic channel at the primary cilium, but not at the plasma membrane ${ }^{25,26}$. In the Xenopus oocyte, despite obvious targeting of PC2 to the plasma membrane, no significant channel activity could be detected ${ }^{35}$. Similarly, no current was detected at the plasma membrane outside the primary cilium in HEK or COS cells that overexpressed PC2 ${ }^{25,60}$. These findings suggest that wild-type PC2 is in a closed conformation when expressed at the plasma membrane, and becomes active at the primary cilium 25,26

In addition, the open state probability of PC2 is dramatically enhanced by membrane depolarization, as well as by elevated intraciliary calcium, possibly detected by the intracellular EF hand motif ${ }^{25,26}$ (FIG. 1b and 4c-d). Under physiological conditions, cation influx (mostly potassium within the collecting duct) through PC2 is predicted to depolarize the primary cilium, although with a 
limited impact on the whole cell potential as the membrane resistance of collecting duct cells is low 26. Extracellular calcium blocks permeation of monovalent cations through $\mathrm{PC2}$, presumably by interacting with Asp643 within the selectivity filter $\left(\mathrm{IC}_{50}=17 \mathrm{mM}\right){ }^{25,34}$ (FIGs. 1d and 1e). However, since urinary concentration of calcium is highly variable, ranging from 5 to $20 \mathrm{mM}, \mathrm{PC} 2$ is likely to be mostly active when urinary calcium concentration is in the lower range.

By contrast, intracellular calcium binding to the EF hand motif within the cytosolic carboxyterminal domain of PC2 is thought to promote channel activation (FIG. 1b) ${ }^{45,46,49}$. Since the resting membrane potential of primary cilia is $-18 \mathrm{mV}$ and intraciliary calcium concentration is estimated to be in the range of $700 \mathrm{nM}{ }^{30}, \mathrm{PC} 2$ is predicted to be in the closed conformation under basal conditions as the intracellular calcium is too low and the membrane is not sufficiently depolarized to mediate channel opening ${ }^{26}$ (FIG. 4c). Thus, additional stimuli such as the stimulation of a membrane receptor and/or the presence of an intracellular second messenger or specific lipids might be required for the activation of PC2 and channel opening within the primary cilium. For example, PC2 might function as an epidermal growth factor (EGF)-activated ion channel ${ }^{56}$. EGF activates PC2 by releasing a protein diaphanous homolog 1 (mDIA1) block at negative membrane potentials ${ }^{61}$. Of note, in mIMCD3 cells, the number of active channels per cilium was relatively low; $66 \%$ of cilia did not show PC2 activity and in the remaining cilia, only one to six channels were active at the primary cilium ${ }^{26}$.

\section{[H2] Mechanosensing by the primary cilium}

Initial reports claimed that PC1 and PC2 mediate mechanosensation at the primary cilium of kidney epithelial cells ${ }^{27}$. In response to shear stress (that is elevated fluid flow), an increase in intracellular calcium was detected in cells that naturally express PC1 and PC2; remarkably, this was not observed in cells that lacked PC1 ${ }^{27}$, nor following inhibition of PC2 with antibodies. The conclusion was that the PC1-PC2 complex was required for fluid flow sensing by the primary cilium in renal tubular epithelial cells ${ }^{27,62}$. In addition, it was suggested that loss of mechanosensation by the primary cilium upon pathogenic mutation of PC1 or PC2 might contribute to cystogenesis ${ }^{27}$ subsequent findings challenged this interesting hypothesis. One study showed that TRPV4, another TRP family channel subunit, interacts with PC2 at the primary cilium and is also critically required for flow sensing, as visualized by calcium imaging ${ }^{10}$. However, since zebrafish or mice that are deficient in TRPV4 do not develop renal cysts, it seems unlikely that defective flow sensing by the primary cilium alone has a causative role in ADPKD ${ }^{10}$.

The proposed role of primary cilia as a calcium-responsive mechanosensor was challenged by one study that used cultured cells derived from a transgenic mouse line that selectively expresses a genetically-encoded ratiometric calcium indicator [G] in all primary cilia ${ }^{30}$. The researchers reported 
that cilia-specific calcium influx did not occur in response to fluid flow, even when supraphysiological fluid flows were applied ${ }^{30}$. These results suggest that mechanosensation by the primary cilium might be independent of ciliary calcium signaling ${ }^{30}$; however, potential technical issues such as oversaturation of the calcium reporter due to a relatively high calcium concentration in the primary cilium might complicate the interpretation of these data ${ }^{63}$. In fact, several other reports support the increase in ciliary calcium in response to mechanical stimuli in cultured renal cells ${ }^{64-67}$.

Another hypothesis that warrants further investigation suggests that potassium and sodium permeability through PC2, independently of a calcium influx ${ }^{25}$, could contribute to flow sensing by the primary cilium and that disruption of this mechanism might have a role in ADPKD. PC2 activation might also be secondary to the opening of a neighboring depolarizing and/or calcium-permeable ciliary channel or plasma membrane channel, such as Piezo $1^{68-70}$, in response to shear stress. In the context of vertebrate left-right asymmetry determination during embryonic development, PC2 might also have a role in cilia mechanosensation, independently of PC1 (BOX 2).

Interestingly, in comparison to wild-type cells, endothelial cells that lack PC1 release less nitric oxide (NO), a vasorelaxing factor, in response to shear stress ${ }^{29}$. Of note, patients with ADPKD suffer from hypertension, a vascular dysfunction that might precede kidney failure ${ }^{71}$. However, shear stress-induced activation of the endothelium seems to depend on the activation of the mechanosensitive ion channel Piezo1 at the plasma membrane ${ }^{69,70}$. Deletion of Piezo1 not only prevents activation of the endothelium in response to shear stress but also causes vascular and valvular developmental defects, as well as impaired NO release and associated hypertension in adult mice ${ }^{69,70,72,73}$. Interestingly, polycystins might regulate the function of Piezo1. One study showed that Pkd1 deletion in arterial myocytes impairs activation of Piezo1-dependent stretch-activated cationic channels at the plasma membrane ${ }^{60}$. Moreover, in both vascular and renal cells, the PC1:PC2 ratio influences Piezo1 activity, at least partly through a mechanoprotection [G] mechanism mediated by the filamin A-actin cytoskeleton network ${ }^{15,60}$; in the absence of PC1 (or when PC2 is elevated) the cortical skeleton becomes stiffer which inhibits opening of Piezo1 induced by membrane stretch. Thus, the impaired shear stress response of renal and endothelial cells in PKD1-knockout mice might be explained by the resulting blunted activation of Piezo1, rather than the loss of a direct contribution of polycystins to the primary cilium mechanosensation ${ }^{27,29}$. Of note, kidney-specific Piezo1-knockout mice do not have a cystic phenotype ${ }^{74}$.

\section{[H2] Calcium release through PC2 at the ER}

The $\mathrm{C}$ terminal domain of PC2 includes an ER retention signal. Accordingly, the majority of PC2 is found at the ER membrane ${ }^{39}$ where it forms a calcium-activated channel that is permeable to 
divalent cations, including $\mathrm{Ca}^{2+}$ and $\mathrm{Ba}^{2+39}$ (FIG. 4d). When PC2 is overexpressed in the LLC-PK1 kidney proximal tubule cell line, vasopressin-induced calcium responses are greatly enhanced, whereas the pathogenic loss-of-function mutant PC2-D511V showed no effect ${ }^{39}$. These findings indicate that PC2 at the ER membrane is permeant to divalent cations ${ }^{14,39,44,75}$ (FIG. 4d). The open state probability of PC2 in response to cytosolic calcium concentrations follows a normal distribution pattern that peaks at $0.3 \mu \mathrm{M}^{44-46,49}$, meaning that channel activity is potentiated at calcium concentrations below 0.3 $\mu \mathrm{M}$, but becomes inhibited at higher concentrations (FIG. 4d $)^{39,44}$. Interestingly, the calcium sensitivity of ER PC2 is modulated by casein kinase phosphorylation of Ser812 within its cytosolic Cterminal domain ${ }^{44}$; the Ser812Ala mutant is approximately 10 -fold less sensitive to calcium activation than the wild-type PC2. In line with these findings, the Ser812Ala mutant greatly reduces potentiation of the vasopressin response by $\mathrm{PC} 2{ }^{44}$. One study demonstrated that at the ER membrane, inositol-1,4,5-trisphosphate receptor $\left(\operatorname{InsP}_{3} \mathrm{R}\right)$-mediated release of $\mathrm{Ca}^{2+}$ from the ER induces opening of the PC2 channel (calcium-induced calcium release), which in turn amplifies the $\mathrm{Ca}^{2+}$ release induced by $\operatorname{InsP}_{3}{ }^{75}$ (FIG. 4d). When calcium is released, the ER lumen becomes negatively charged and a potassium influx is required to balance the positive charge deficit; whether PC2 also has a role in counterion conductance at the ER membrane and indirectly influences calcium release through InsP3Rs has not yet been explored. Collectively, these findings suggest that the selectivity, and possibly the gating, of PC2 might differ depending on its subcellular localization (that is, primary cilium, plasma membrane or ER), as location might determine, for example, which lipids are available for binding to polycystins ${ }^{34}$, as well as other potential binding partners, such as other TRP channels, including TRPV4 and TRPC1 ${ }^{10,11}$. The potential modulation of polycystin functions by subcellular localization needs to be further evaluated. At this stage, it is also unclear whether PC2 at the ER requires PC1, or another related molecule, to function. Moreover, whether PC2 at the ER is relevant to ADPKD remains to be examined.

\section{[H1] Conclusions and perspectives}

PC2 is a non-selective cationic channel, which is activated by both membrane depolarization and intracellular ciliary calcium, and inhibited by high extracellular calcium ${ }^{25,26}$. PC2 localizes to the primary cilium and ER membrane, and its ability to permeate calcium might be conditioned by its subcellular localization; this effect might be due to the specific membrane environment and/or the presence and contribution of auxiliary proteins such as PC1, fibrocystin, PC1-like proteins, PC2-like proteins or other TRP subunits. PC2 might also have a non-channel function, for instance in mitotic spindles, with potential effects on cell division, or even as a negative regulator of other types of ion channels, including the ryanodine receptor at the ER ${ }^{12}, \mathrm{~K}_{2 \mathrm{P}}$ TREK channels ${ }^{76}$ and Piezo1 ${ }^{15,60}$ at the 
plasma membrane. Breakthroughs in the structural characterization of PC1-PC2 revealed that PC1 might occlude the PC2 ion channel pore, suggesting that ion channel permeation might be unrelated to ADPKD; this hypothesis is reinforced by the observation that no pathogenic mutations are found in the putative pore region of PC1 ${ }^{33}$ (FIG. 1b). However, these findings come from a single report and await confirmation; importantly, they need to be functionally validated by electrophysiological data. Nonetheless, these unexpected observations raise important novel questions: Is the solved PC1-PC2 structure physiologically relevant? What is the function of the homomeric PC2 channel, besides its recognized role in nodal cells during embryonic development? What mediates PC2 calcium permeability in the ER? If channel activity is unrelated to ADPKD, what is the function of the PC1-PC2 heteromeric complex and how do pathogenic mutations cause the disease? Since numerous mutations are found in the PCD of both PC1 and PC2, what is the role of this extracellular 'lid' that sits on top of the channel complex? Is the PCD involved in either physical and/or chemical activation of the heteromeric PC1-PC2 complex? What function might the PC1 PLAT domain fulfill? Which lipids bind to PC1-PC2 and how does this may affect its function or relate to ADPKD?

Despite being discovered more than 20 years ago, the biological role of polycystins and how mutations in PKD1 and PKD2 cause ADPKD is still obscure and requires substantial further work to enable the identification of potential therapeutic options.

\section{References:}

1. Harris, P.C. \& Torres, V.E. Polycystic Kidney Disease. Annu Rev Med 60, 321-337 (2009).

2. Arnaout, M.A. Molecular genetics and pathogenesis of autosomal dominant polycystic kidney disease. Annu Rev Med 52, 93-123 (2001).

3. Torres, V.E. \& Harris, P.C. Mechanisms of Disease: autosomal dominant and recessive polycystic kidney diseases. Nat Clin Pract Nephrol 2, 40-55; quiz 55 (2006).

4. Patel, A. \& Honore, E. Polycystins and renovascular mechanosensory transduction. Nat Rev Nephrol 6, 530-538 (2010).

5. Hughes, J., et al. The polycystic kidney disease 1 (PKD1) gene encodes a novel protein with multiple cell recognition domains. Nat Genet 10, 151-160 (1995).

6. Mochizuki, T., et al. PKD2, a gene for polycystic kidney disease that encodes an integral membrane protein. Science 272, 1339-1342 (1996).

7. Wilson, P.D. Polycystic kidney disease. N Engl J Med 350, 151-164 (2004).

8. Kim, I., et al. Polycystin-2 Expression Is Regulated by a PC2-binding Domain in the Intracellular Portion of Fibrocystin. J Biol Chem 283, 31559-31566 (2008).

9. Outeda, P., et al. A novel model of autosomal recessive polycystic kidney questions the role of the fibrocystin C-terminus in disease mechanism. Kidney Int 92, 1130-1144 (2017).

10. Kottgen, M., et al. TRPP2 and TRPV4 form a polymodal sensory channel complex. J Cell Biol 182, 437-447 (2008).

This study shows that PC2 interacts with another TRP channel subunit to form an heteromer 
11. Kobori, T., Smith, G.D., Sandford, R. \& Edwardson, J.M. The transient receptor potential channels TRPP2 and TRPC1 form a heterotetramer with a 2:2 stoichiometry and an alternating subunit arrangement. J Biol Chem 284, 35507-35513 (2009).

This work demonstrates an interaction between PC2 and TRPC1

12. Anyatonwu, G.I., Estrada, M., Tian, X., Somlo, S. \& Ehrlich, B.E. Regulation of ryanodine receptor-dependent calcium signaling by polycystin-2. Proc Natl Acad Sci U S A 104, 64546459 (2007).

13. Li, Y., et al. Polycystin-1 interacts with inositol 1,4,5-trisphosphate receptor to modulate intracellular Ca2+ signaling with implications for polycystic kidney disease. J Biol Chem 284, 36431-36441 (2009).

This study indicates that PC2 allows the amplification of the $I P_{3}$-dependent calcium release from the ER

14. Li, Y., Wright, J.M., Qian, F., Germino, G.G. \& Guggino, W.B. Polycystin 2 interacts with type I inositol 1,4,5-trisphosphate receptor to modulate intracellular Ca2+ signaling. J Biol Chem 280, 41298-41306 (2005).

15. Peyronnet, R., et al. Piezo1-dependent stretch-activated channels are inhibited by Polycystin2 in renal tubular epithelial cells. EMBO Rep 14, 1143-1148 (2013).

Evidence that PC2 inhibits Piezo1 opening in renal epithelial cells trhough a cytoskeleton-mediated mechanoprotection mechanism

16. Lantinga-van Leeuwen, I.S., et al. Lowering of $\mathrm{Pkd} 1$ expression is sufficient to cause polycystic kidney disease. Hum Mol Genet 13, 3069-3077 (2004).

This study demonstrates that an hypomorphic effect on the Pkd1 somatic allele is sufficient to cause ADPKD

17. Piontek, K., Menezes, L.F., Garcia-Gonzalez, M.A., Huso, D.L. \& Germino, G.G. A critical developmental switch defines the kinetics of kidney cyst formation after loss of Pkd1. Nat Med 13, 1490-1495 (2007).

This report shows that loss of $P$ kd1 1 before post-natal day 13 results in severely cystic kidneys

18. Wu, G., et al. Somatic inactivation of Pkd2 results in polycystic kidney disease. Cell 93, 177188 (1998).

19. Delmas, P. Polycystins: from mechanosensation to gene regulation. Cell 118, 145-148 (2004).

20. Choi, Y.H., et al. Polycystin-2 and phosphodiesterase $4 \mathrm{C}$ are components of a ciliary A-kinase anchoring protein complex that is disrupted in cystic kidney diseases. Proc Natl Acad Sci U S A 108, 10679-10684 (2011).

21. Yamaguchi, T., Hempson, S.J., Reif, G.A., Hedge, A.M. \& Wallace, D.P. Calcium restores a normal proliferation phenotype in human polycystic kidney disease epithelial cells. J Am Soc Nephrol 17, 178-187 (2006).

22. Yamaguchi, T., et al. Calcium restriction allows CAMP activation of the B-Raf/ERK pathway, switching cells to a cAMP-dependent growth-stimulated phenotype. J Biol Chem 279, 4041940430 (2004).

23. Hanaoka, K., et al. Co-assembly of polycystin-1 and -2 produces unique cation-permeable currents. Nature 408, 990-994 (2000).

This study demonstrates that, in transfected CHO cells, PC1 interacts with PC2 at the plasma membrane to form a cationic channel

24. Delmas, P., et al. Gating of the polycystin ion channel signaling complex in neurons and kidney cells. Faseb J 18, 740-742 (2004).

This report demonstrates that, when overexpressed in sympathetic neurons, the PC1-PC2 complex can be activated at the plasma membrane by a PC1 targeting antibody

25. Liu, X., et al. Polycystin-2 is an essential ion channel subunit in the primary cilium of the renal collecting duct epithelium. Elife 7(2018).

This research provides evidence by patch clamp recordings of PC2 opening at the primary cilium of renal cells 
26. Kleene, S.J. \& Kleene, N.K. The native TRPP2-dependent channel of murine renal primary cilia. Am J Physiol Renal Physiol 312, F96-F108 (2017).

This study demonstrates that PC2 at the primary cilium is activated by both depolarization and an increase in intraciliary calcium

27. Nauli, S.M., et al. Polycystins 1 and 2 mediate mechanosensation in the primary cilium of kidney cells. Nat Genet 33, 129-137 (2003).

This report shows that the PC1-PC2 complex mediates flow-dependent activation of the primary cilium in renal cells

28. Aboualaiwi, W.A., et al. Ciliary Polycystin-2 Is a Mechanosensitive Calcium Channel Involved in Nitric Oxide Signaling Cascades. Circ Res 104, 860-869 (2009).

29. Nauli, S.M., et al. Endothelial cilia are fluid shear sensors that regulate calcium signaling and nitric oxide production through polycystin-1. Circulation 117, 1161-1171 (2008).

This research demonstrates that activation of PC1-PC2 by shear stress increases the release of NO by the endothelium

30. Delling, M., et al. Primary cilia are not calcium-responsive mechanosensors. Nature 531, 656660 (2016).

This study shows that stimulation of the primary cilium by flow does not induce an increase in intraciliary calcium

31. Shen, P.S., et al. The Structure of the Polycystic Kidney Disease Channel PKD2 in Lipid Nanodiscs. Cell 167, 763-773 e711 (2016).

Report of the first structural determination of PC2 by cryo-EM

32. Grieben, M., et al. Structure of the polycystic kidney disease TRP channel Polycystin-2 (PC2). Nat Struct Mol Biol 24, 114-122 (2017).

This study provides evidence that a large polycystin domain sits on top of the PC2 channel and is mutated in ADPKD

33. Su, Q., et al. Structure of the human PKD1/PKD2 complex. Science (2018).

Report of the first structural determination of the PC1-PC2 complex

34. Wilkes, M., et al. Molecular insights into lipid-assisted $\mathrm{Ca}(2+)$ regulation of the TRP channel Polycystin-2. Nat Struct Mol Biol 24, 123-130 (2017).

35. Zheng, W., et al. Hydrophobic pore gates regulate ion permeation in polycystic kidney disease 2 and 2L1 channels. Nat Commun 9, 2302 (2018).

This study shows that Leu777 in the S6 of PC2 acts as an hydrophobic gate

36. Delmas, P., et al. Polycystins, calcium signaling, and human diseases. Biochem Biophys Res Commun 322, 1374-1383 (2004).

37. Harris, P.C. \& Torres, V.E. Genetic mechanisms and signaling pathways in autosomal dominant polycystic kidney disease. J Clin Invest 124, 2315-2324 (2014).

38. Qian, F., et al. PKD1 interacts with PKD2 through a probable coiled-coil domain. Nat Genet 16, 179-183 (1997).

39. Koulen, P., et al. Polycystin-2 is an intracellular calcium release channel. Nat Cell Biol 4, 191197 (2002).

This research demonstrates that PC2 at the ER membrane is a calcium release channel

40. Kip, S.N., et al. [Ca2+]i reduction increases cellular proliferation and apoptosis in vascular smooth muscle cells: relevance to the ADPKD phenotype. Circ Res 96, 873-880 (2005).

41. Clapham, D.E. TRP channels as cellular sensors. Nature 426, 517-524 (2003).

42. Hulse, R.E., Li, Z., Huang, R.K., Zhang, J. \& Clapham, D.E. Cryo-EM structure of the polycystin 2-I1 ion channel. Elife 7(2018).

43. Su, Q., et al. Cryo-EM structure of the polycystic kidney disease-like channel PKD2L1. Nat Commun 9, 1192 (2018).

44. Cai, Y., et al. Calcium dependence of polycystin-2 channel activity is modulated by phosphorylation at Ser812. J Biol Chem 279, 19987-19995 (2004).

45. Celic, A.S., et al. Calcium-induced conformational changes in C-terminal tail of polycystin-2 are necessary for channel gating. J Biol Chem 287, 17232-17240 (2012). 
46. Kuo, I.Y., et al. The number and location of EF hand motifs dictates the calcium dependence of polycystin-2 function. FASEB J 28, 2332-2346 (2014).

47. Allen, M.D., Qamar, S., Vadivelu, M.K., Sandford, R.N. \& Bycroft, M. A high-resolution structure of the EF-hand domain of human polycystin-2. Protein Sci 23, 1301-1308 (2014).

48. Schumann, F., et al. Ca2+-dependent conformational changes in a C-terminal cytosolic domain of polycystin-2. J Biol Chem 284, 24372-24383 (2009).

49. Petri, E.T., et al. Structure of the EF-hand domain of polycystin-2 suggests a mechanism for Ca2+-dependent regulation of polycystin-2 channel activity. Proc Natl Acad Sci U S A 107, 9176-9181 (2010).

50. Hofherr, A., Wagner, C., Fedeles, S., Somlo, S. \& Kottgen, M. N-glycosylation determines the abundance of the transient receptor potential channel TRPP2. J Biol Chem 289, 14854-14867 (2014).

51. Giamarchi, A., et al. The versatile nature of the calcium-permeable cation channel TRPP2. EMBO Rep 7, 787-793 (2006).

52. Arif Pavel, M., et al. Function and regulation of TRPP2 ion channel revealed by a gain-offunction mutant. Proc Natl Acad Sci U S A 113, E2363-2372 (2016).

53. $\mathrm{Yu}, \mathrm{S}$., et al. Essential role of cleavage of Polycystin-1 at G protein-coupled receptor proteolytic site for kidney tubular structure. Proc Natl Acad Sci U S A 104, 18688-18693 (2007).

54. Yu, Y., et al. Structural and molecular basis of the assembly of the TRPP2/PKD1 complex. Proc Natl Acad Sci U S A (2009).

55. Kim, S., et al. The polycystin complex mediates Wnt/Ca(2+) signalling. Nat Cell Biol 18, 752764 (2016).

This report shows that Wnt binds to PC1 and activates PC2 in the complex

56. Ma, R., et al. PKD2 functions as an epidermal growth factor-activated plasma membrane channel. Mol Cell Biol 25, 8285-8298 (2005).

This study provides evidence that EGF activates PC2 currents in renal cells

57. Harris, P.C., et al. Cyst number but not the rate of cystic growth is associated with the mutated gene in autosomal dominant polycystic kidney disease. J Am Soc Nephrol 17, 30133019 (2006).

58. Raychowdhury, M.K., et al. Characterization of single channel currents from primary cilia of renal epithelial cells. J Biol Chem 280, 34718-34722 (2005).

59. Geng, L., et al. Polycystin-2 traffics to cilia independently of polycystin-1 by using an Nterminal RVxP motif. J Cell Sci 119, 1383-1395 (2006).

This report demonstrates that PC2 is targeted to the primary cilium independently of PC1

60. Sharif Naeini, R., et al. Polycystin-1 and -2 dosage regulates pressure sensing. Cell 139, 587596 (2009).

This study shows that the PC1:PC2 ratio regulates the opening of the Piezo1-dependent mechanosensitive channels in arterial myocytes

61. Bai, C.X., et al. Activation of TRPP2 through mDia1-dependent voltage gating. Embo J 27, 1345-1356 (2008).

62. Nauli, S.M. \& Zhou, J. Polycystins and mechanosensation in renal and nodal cilia. Bioessays 26, 844-856 (2004).

63. Nauli, S.M., Pala, R. \& Kleene, S.J. Calcium channels in primary cilia. Curr Opin Nephrol Hypertens 25, 452-458 (2016).

64. Lee, K.L., et al. The primary cilium functions as a mechanical and calcium signaling nexus. Cilia 4, 7 (2015).

65. Jin, X., et al. Cilioplasm is a cellular compartment for calcium signaling in response to mechanical and chemical stimuli. Cell Mol Life Sci 71, 2165-2178 (2014).

66. Su, S., et al. Genetically encoded calcium indicator illuminates calcium dynamics in primary cilia. Nat Methods 10, 1105-1107 (2013). 
67. Yuan, S., Zhao, L., Brueckner, M. \& Sun, Z. Intraciliary calcium oscillations initiate vertebrate left-right asymmetry. Curr Biol 25, 556-567 (2015).

68. Coste, B., et al. Piezo1 and Piezo2 are essential components of distinct mechanically activated cation channels. Science 330, 55-60 (2010).

Report on the discovery of the mechanosensitive Piezo channels

69. Ranade, S.S., et al. Piezo1, a mechanically activated ion channel, is required for vascular development in mice. Proc Natl Acad Sci U S A 111, 10347-10352 (2014).

70. Li, J., et al. Piezo1 integration of vascular architecture with physiological force. Nature 515, 279-282 (2014).

71. Bichet, D., Peters, D., Patel, A.J., Delmas, P. \& Honore, E. Cardiovascular polycystins: insights from autosomal dominant polycystic kidney disease and transgenic animal models. Trends Cardiovasc Med 16, 292-298 (2006).

72. Wang, S., et al. Endothelial cation channel PIEZO1 controls blood pressure by mediating flowinduced ATP release. J Clin Invest 126, 4527-4536 (2016).

This study shows that opening of Piezo1 mediates flow-dependent arterial dilation

73. Nonomura, K., et al. Mechanically activated ion channel PIEZO1 is required for lymphatic valve formation. Proc Natl Acad Sci U S A 115, 12817-12822 (2018).

74. Martins, J.R., et al. Piezo1-dependent regulation of urinary osmolarity. Pflugers Arch 468, 1197-1206 (2016).

75. Sammels, E., et al. Polycystin-2 activation by inositol 1,4,5-trisphosphate-induced Ca2+ release requires its direct association with the inositol 1,4,5-trisphosphate receptor in a signaling microdomain. J Biol Chem (2010).

This research demonstrates that opening of PC2 at the ER membrane amplifies the release of calcium through the Ins $P_{3} R$

76. Peyronnet, R., et al. Mechanoprotection by Polycystins against Apoptosis Is Mediated through the Opening of Stretch-Activated K(2P) Channels. Cell Rep 1, 241-250 (2012).

This study provides evidence that PC2 inhibits Piezo1 opening by membrane stretch

77. Shen, P.S. The 2017 Nobel Prize in Chemistry: cryo-EM comes of age. Anal Bioanal Chem 410, 2053-2057 (2018).

78. McGrath, J., Somlo, S., Makova, S., Tian, X. \& Brueckner, M. Two populations of node monocilia initiate left-right asymmetry in the mouse. Cell 114, 61-73 (2003).

79. Pennekamp, P., et al. The ion channel polycystin-2 is required for left-right axis determination in mice. Curr Biol 12, 938-943 (2002).

This report shows that loss of Pkd2 causes embryonic laterality defects

80. Yoshiba, S., et al. Cilia at the Node of Mouse Embryos Sense Fluid Flow for Left-Right Determination via Pkd2. Science 338, 226-231 (2012).

This study demonstrates that flow-induced PC2 opening in nodal cells is responsible for left-right asymmetry

81. Karcher, C., et al. Lack of a laterality phenotype in Pkd1 knock-out embryos correlates with absence of polycystin-1 in nodal cilia. Differentiation 73, 425-432 (2005).

82. Field, S., et al. Pkd111 establishes left-right asymmetry and physically interacts with Pkd2. Development 138, 1131-1142 (2011).

83. Vogel, P., et al. Situs inversus and related ciliopathies in Dpcd-/-, Pkd1/1-/- and Nme7-/- mice. Vet Pathol (2009).

84. Vogel, P., et al. Situs inversus in Dpcd/Poll-/-, Nme7-/- , and Pkd1/1-/- mice. Vet Pathol 47, 120-131 (2010).

85. Vetrini, F., et al. Bi-allelic Mutations in PKD1L1 Are Associated with Laterality Defects in Humans. Am J Hum Genet 99, 886-893 (2016).

86. Kamura, K., et al. Pkd1/1 complexes with Pkd2 on motile cilia and functions to establish the left-right axis. Development 138, 1121-1129 (2011). 
87. Grimes, D.T., et al. Genetic Analysis Reveals a Hierarchy of Interactions between PolycystinEncoding Genes and Genes Controlling Cilia Function during Left-Right Determination. PLoS Genet 12, e1006070 (2016).

\section{Acknowledgements:}

The authors thank the Human Frontier Science Program, the Fondation pour la Recherche Médicale and the Agence Nationale de la Recherche for support.

\section{Author contributions:}

E.H. wrote the manuscript, D.D. performed the structural modeling and A.P. edited the manuscript.

\section{Related links}

http://pkdb.mayo.edu

http://www.holeprogram.org

\section{Key points}

- The channel activity of PC2 at the primary cilium of renal collecting duct cells is independent of PC1.

- Opening of PC2 is controlled by an internal hydrophobic gate; it is enhanced by membrane depolarization and an increase in intraciliary calcium.

- $\quad$ PC1 and PC2 assemble in a 1:3 ratio in the PC1-PC2 complex.

- PC1 might prevent cation permeation through the heteromeric PC1-PC2 complex by occluding the pore with three positively charged residues.

- Extracellular polycystin domains in PC1 and PC2 are hot spots for pathogenic mutations.

\section{BOX 1: Cryo-electron microscopy}

Crystallization of transmembrane proteins in well-ordered crystals suitable for X-ray crystallography remains a difficult task. As an alternative approach, cryo-electron microscopy (cryo-EM) is a combination of methods that enable the creation of 3D protein models using focused beams of electrons and super-cold temperatures ${ }^{77}$. The protein is frozen in a thin, single-molecule thick, layer of glass-like ice that is then bombarded with electrons. An advantage of the freezing process is that it preserves proteins and complexes in their native state. In addition, for the study of membrane proteins in their nearly native lipid bilayer environment, the transmembrane domain can be 
stabilized with lipid nanodiscs. The irradiation of these nanodiscs with low-energy electrons produces 2D images of individual protein particles in many orientations. Then, cryo-EM methods sort and average hundreds of thousands of these images to build a 3D map and computationally construct a 3D model of the protein. Cryo-EM is an expanding structural biology technique that allows highresolution ( $\sim 3$ to $5 \AA$ ) structural determination of large biomolecules and thus complements other structural biology techniques such as X-ray crystallography and nuclear magnetic resonance (NMR).

\section{BOX 2: PC2 and the determination of left-right asymmetry}

Although vertebrates show a bilaterally symmetrical body-plan, visceral organs show left-right asymmetry in their structure and position, which is best exemplified by the heart. Symmetries are broken during early development, in a process that involves an unidirectional fluid flow, termed nodal flow, within an embryonic cavity at the ventral midline ${ }^{78,79}$; PC2 is exclusively expressed in the crown cells at the edge of the ventral node ${ }^{80}$. Nodal flow generated by the rotational movement of motile node cell monocilia is detected by the non-motile perinodal sensory monociliated cells ${ }^{78-80}$. Leftward nodal flow is responsible for breaking left-right symmetry in the embryo through activation of PC2 in crown cells ${ }^{80}$. Accordingly, homozygous PC2 mutant embryos show laterality defects ${ }^{79}$. Notably, PC1 is not expressed in nodal crown cells ${ }^{81}$, further indicating that PC2 can operate independently of $\mathrm{PC}^{25}{ }^{25}$; moreover left-right asymmetry is unaffected in Pkd1-knockout mice ${ }^{81}$. By contrast, a laterality defect is also observed in a PC1-like 1 protein loss-of-function mutant and in PKD1L1\% mouse models, as well as in humans carrying mutations in this gene ${ }^{82-85}$. Accordingly, PC1like 1 protein interacts with PC2 and this interaction has a role in flow detection by nodal cells $82,86,87$. When nodal flow occurs, an asymmetric calcium increase is detected at the left margin of the node, upstream of the asymmetrically expression of nodal genes ${ }^{79,80}$. Notably, ciliary localization of PC2 is essential for the control of left-right asymmetry ${ }^{80}$, but whether or not PC2 directly senses nodal flow or molecules transported by unidirectional nodal flow remains an open question ${ }^{30,80}$. 


\section{Figure legends:}

Figure 1: The PC1-PC2 heteromeric complex. a| Topological model of polycystin 1 (PC1) and PC2. PC1 comprises an N-terminal domain (NTD), which includes an intracellular PC1 lipoxygenase and alpha toxin (PLAT) domain between transmembrane helix 1 (TM1) and TM2, followed by a voltage sensor-like domain (VSD) that contains an extracellular polycystin domain (PCD) between segment 1 (S1) and S2. The region indicated by the dashed line between S5 and S6 was not resolved in the PC1 structure. b| Topology of a PC2 subunit, including a VSD and a pore domain between S5 and S6, which includes two pore helices (PH1 and $\mathrm{PH} 2$ ) that surround the selectivity filter (SF). c| Structure of a PC1 subunit. The dashed line represents a 29 amino acid long segment (4051-4080), upstream of S6, which was not resolved in the PC1 structure. In PC2 and in other TRP subunits, this region corresponds to the pore domain. Pathogenic substitution mutations, as described by the ADPKD mutation database, [JA: please add hyperlink: http://pkdb.mayo.edu] are indicated by gray spheres. $d$ l Structure of PC2. Asp643 in the SF is responsible for the binding of extracellular calcium and inhibition of PC2 permeation. Leu677 at the intracellular side of S6 acts as a hydrophobic gate. el Structure of the heteromeric polycystin complex including one PC1 (in blue and red) and three PC2 subunits (in green). For easier visualization, the polycystin domains (PCD) are not represented. Magnification of the pore region shows that positively charged residues Arg4100/Arg4107/His4111 from PC1 occlude the permeation pathway in the PC1-PC2 heteromeric complex. PDB accession number: 6A70; PyMOL 1.3 software.

Figure 2: Closed and open states of PC2. al Wild-type PC2 channel in a closed state. The ionic pore is constricted at the external side of the channel within the selectivity filter between amino acids Leu641-Gly-Asp643. Leu677 forms an internal hydrophobic gate. b| The Phe604Pro gain-of-function mutation, located within S5, constitutively opens PC2. The hydrophobic gate L677 swings away from the ionic path in Phe604Pro but the external constriction at the selectivity filter is not altered. The solvent-accessible pathway is shown as a blue cloud (calculated with the HOLE program) [JA: Please add hyperlink: http://www.holeprogram.org]. PDB accession numbers: 5T4D and 6D1W; PyMOL 1.3 software. 
Figure 3: Proposed mechanistic models for the gating of PC2. a| Closed state of the PC2 channel. The polycystin domain (PCD) sits on top of the channel like a lid. The positively charged S4 segment is a voltage sensor domain (VSD) that changes conformation in response to a change in membrane potential. A $3_{10}$ helix is located at the basis of the $S 4$ and is predicted to be involved in gating movements of the S4-S5 linker. The $\mathbf{S 6}$ helix comprises a $\pi$ helix that acts as a point of flexibility that causes the rotation of the Leu677 hydrophobic gate at the inner side of the S6. The hydrophobic gate Leu677 of S6 blocks permeation of cations. Water $\left(\mathrm{H}_{2} \mathrm{O}\right)$ accumulation in the funnel underneath the polycystin domains (PCDs) might prevent permeation through the selectivity filter that accommodates dehydrated cations. b| Gating model involving a conformational change in the PCD. Dehydration of the lateral funnels between PCD and VSD is thought to control permeation through the pore ${ }^{34}$. Fatty acids (FA) block the lateral funnels and prevent water accumulation, which allows permeation of dehydrated cations through the selectivity filter. The horizontal arrows indicate conformational changes in S6. Although the role of lipids in the regulation of PC2 activation has not yet been functionally demonstrated, lipid-mediated interaction between PCD and VSD, as demonstrated in structural studies, suggests a potential lipid-mediated activation mechanism for $\mathrm{PC} 2{ }^{34} \mathrm{c}$ | Gating model mediated by VSD. Upon depolarization, a conformational change in the positively-charged S4 drives a movement of the S4-S5 linker, possibly coupled to $S 6$ via an hydrogen bond between Gln585 and Lys688, that results in a $\pi$ to $\alpha$ helix conformation change of $S 6$ and opening of the lower hydrophobic gate (Leu677) ${ }^{31,35,43}$. $d$ | The Phe604Pro mutant bends S5, which is predicted to induce a movement of the S4-S5 linker and activate the channel by opening the Leu677 inner gate ${ }^{35}$. 
Figure 4: Ion channel function of PC2 at the primary cilium and the ER. a| Patch clamp recording (inside-out configuration) of PC2 at the primary cilium of renal collecting duct cells. Once the electrode is attached to the primary cilium, the patch is excised in the inside-out configuration; the cilium can then be depolarized and exposed to free intracellular calcium ([free- $\left.\mathrm{Ca}^{2+}\right]_{\text {in }}$ ) concentrations (adapted from ${ }^{25}$ ). b| Inside-out cilium patch record from an inner medullary collecting duct epithelial cells ( $p$ IMCD) cilia (adapted from ${ }^{25}$ ). The patch contained at least three active channels; 01,02 and 03 indicate open states. Cytosolic calcium was progressively elevated from $0.09 \mu \mathrm{M}$ to $0.3 \mu \mathrm{M}$, then to $3 \mu \mathrm{M}$ and finally washed out. c| Relationship between open channel probability and membrane voltage with increasing internal calcium concentrations of ciliary PC2 channels (adapted from ${ }^{26}$ ). $d$ | PC2 opening amplifies the release of calcium through the $\operatorname{InsP}_{3} R$ by a calcium-induced calcium release mechanism ${ }^{39,44,75}$; the PC2 open channel probability over cytosolic calcium concentration is normally distributed ${ }^{44}$.[JA: Licence for reproduction: (FIG. 4a-b) https://creativecommons.org/licenses/by/4.0/]

\section{Glossary terms:}

Primary cilium: single non-motile cilium that lacks a central pair of microtubules present in all mammalian cells, except immune cells.

Permeation: permeability of ions through channels.

Gating: molecular mechanisms of channel opening and closing.

Non-selective cationic channel: a channel permeable to all cations, including sodium, potassium and calcium.

Coiled-coil domain: structural motif in proteins in which 2-7 $\alpha$-helices are coiled together, mediating protein-protein interaction.

Lipid nanodiscs: lipid bilayer mimetics that function as synthetic model membranes in which purified proteins can be reconstituted for structural determination with cryo-EM

Selectivity filter: segment within the pore of an ion channel that controls its ionic permeability

Voltage sensor: charged domain of an ion channel (S4) that confers voltage sensitivity

Cation sink: negative charges present at the extracellular side of the channel that attract cations toward the selectivity filter

Conduction pathway: the pore of the ion channel

Vestibule: the entrance of the ion channel pore

EF hand motif: motif with a helix-loop-helix topology to which calcium ions bind

Amphipathic: contains both hydrophobic and hydrophilic groups 
$\pi$ helix: helix with 4.4 amino acids per turn; $\alpha$ helices have 3.6 amino acids per turn.

Hydrophobic gate: hydrophobic residue that repels water and prevents ion permeation through an ion channel

Electrostatic maps: illustrates in 3D the charge distributions over a protein

Ratiometric calcium indicator: a fluorescence method based on the use of a ratio between two fluorescence intensities (for instance Fura-2 calcium probe)

Mechanoprotection: inhibition of mechano-sensitive ion channels by the cytoskeleton 
Figure 1

a

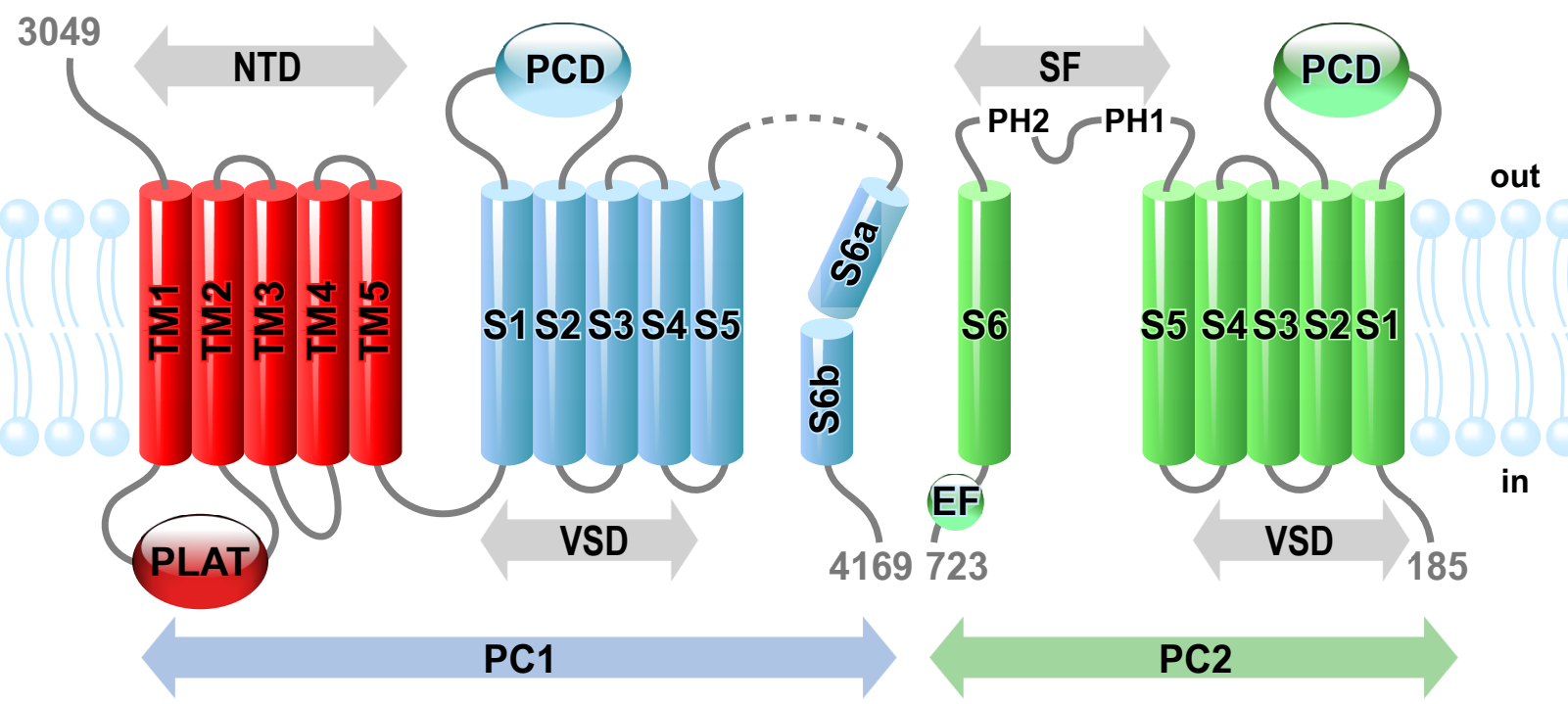

b

d
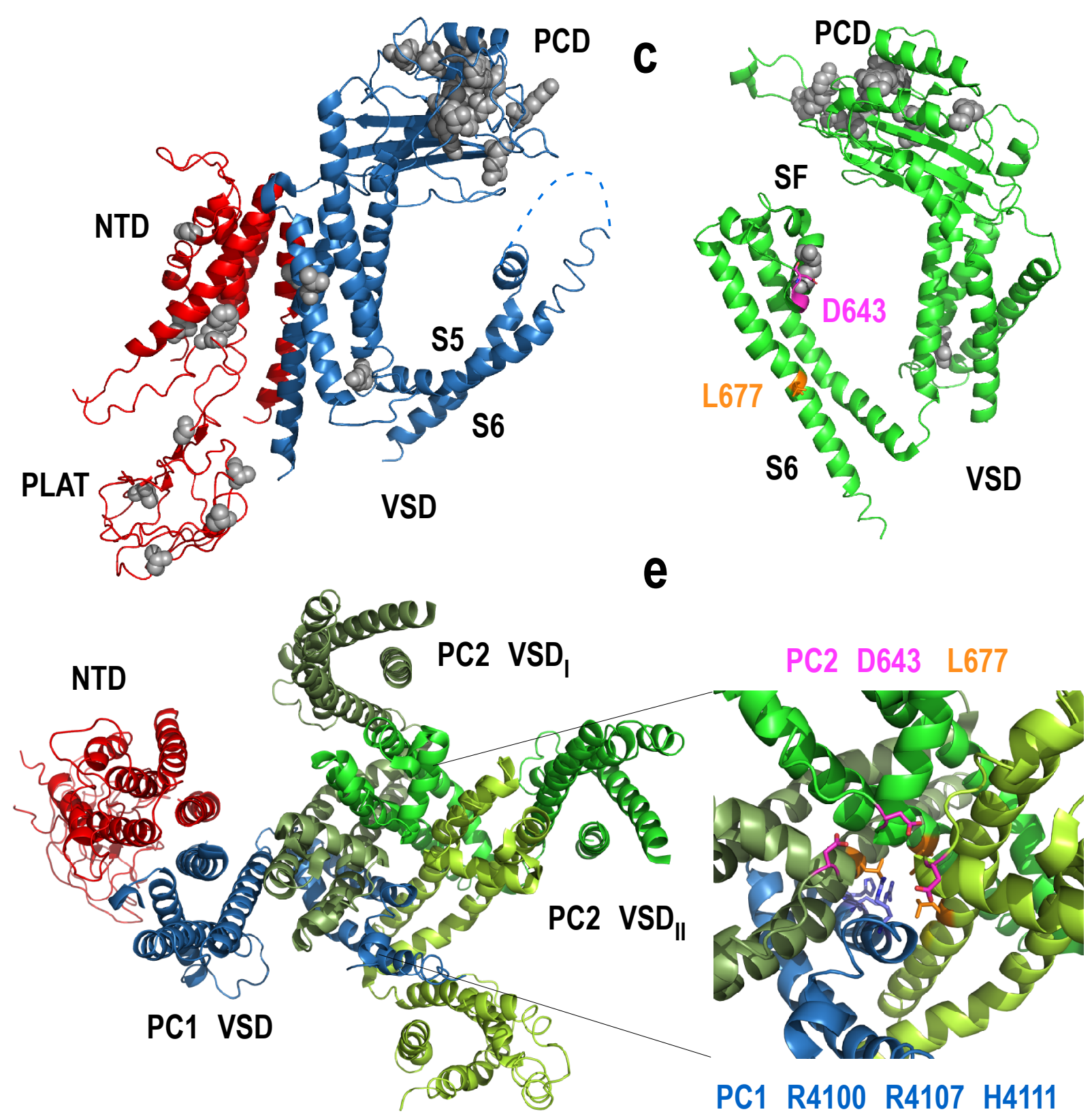

PC2 VSD $_{\text {III }}$ 
Figure 2

PC2 WT

PC2 F604P

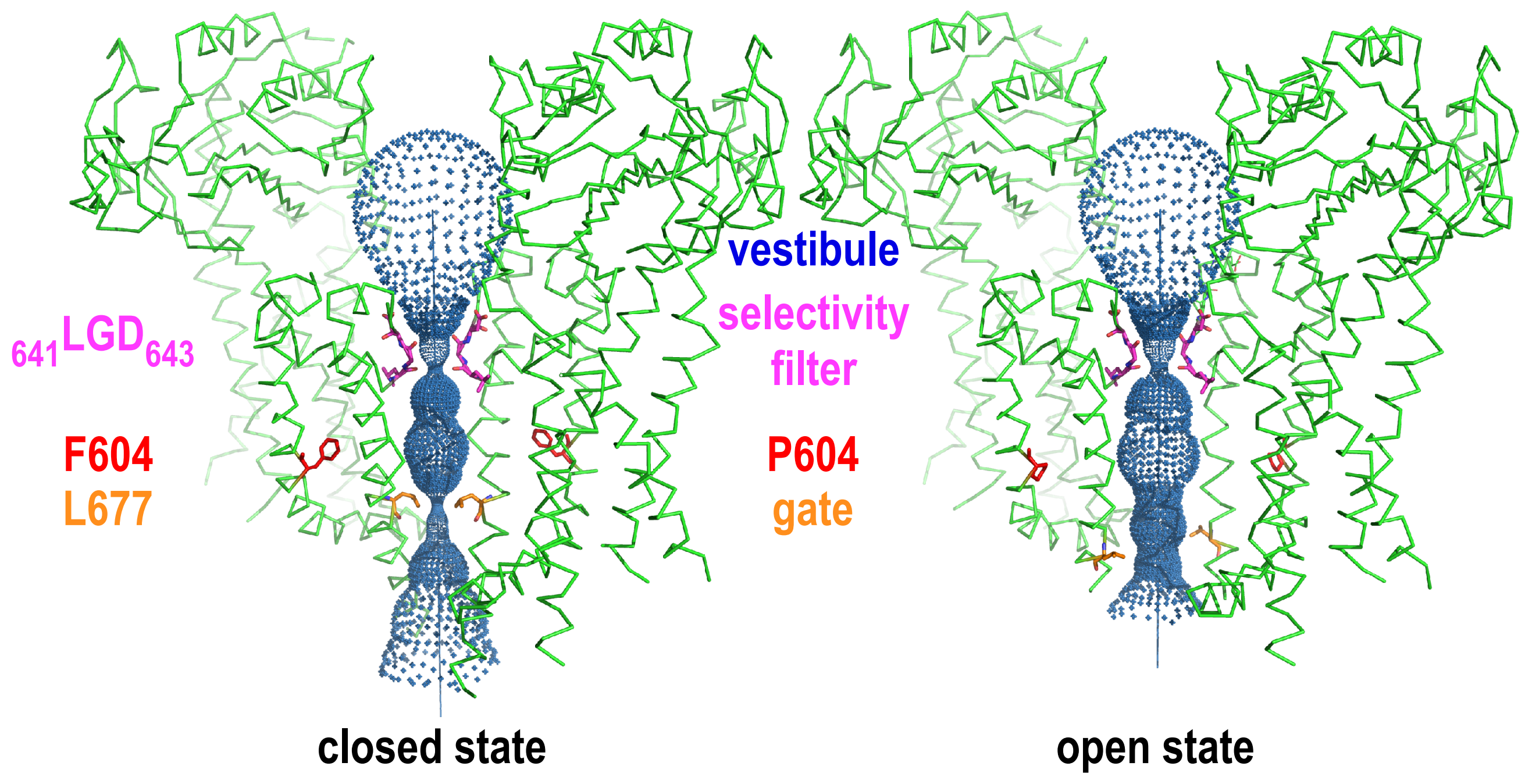


Figure 3

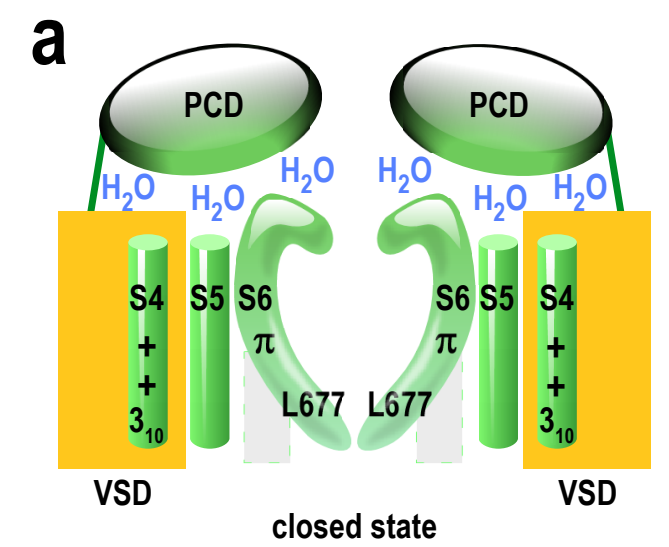

voltage

C PC2 gating by VSD

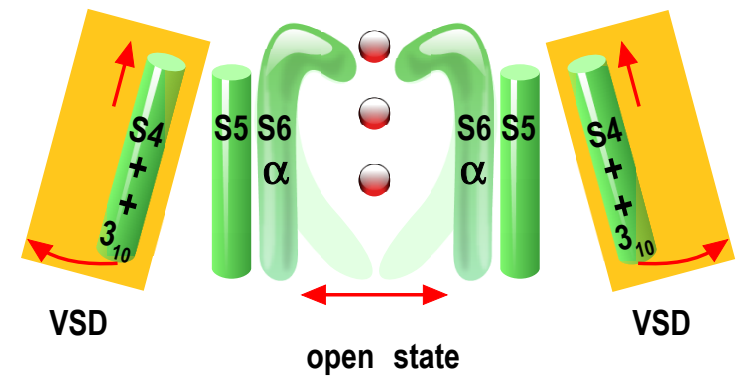

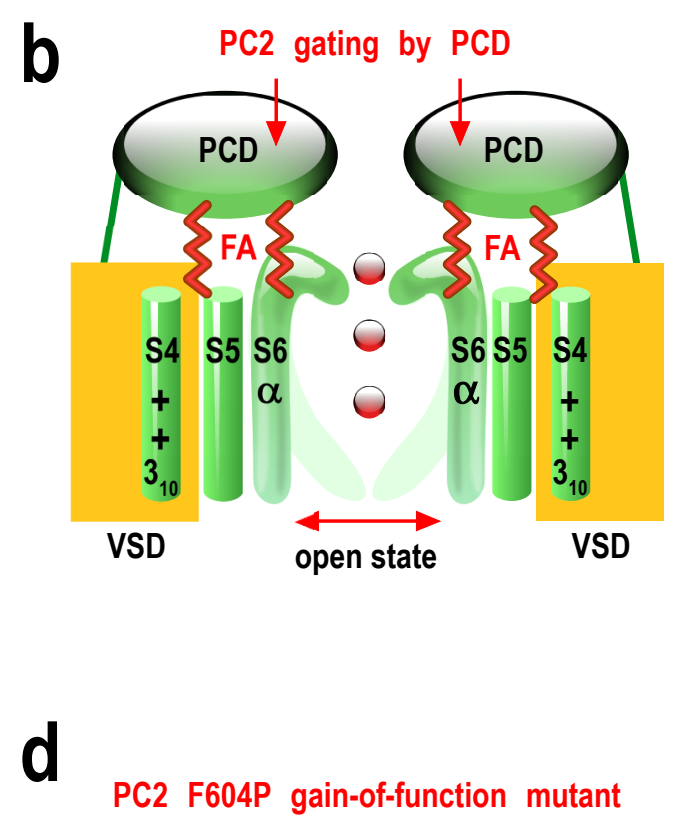

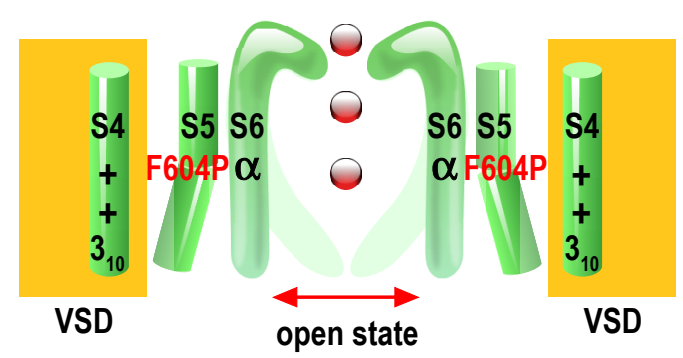


Figure 4

a
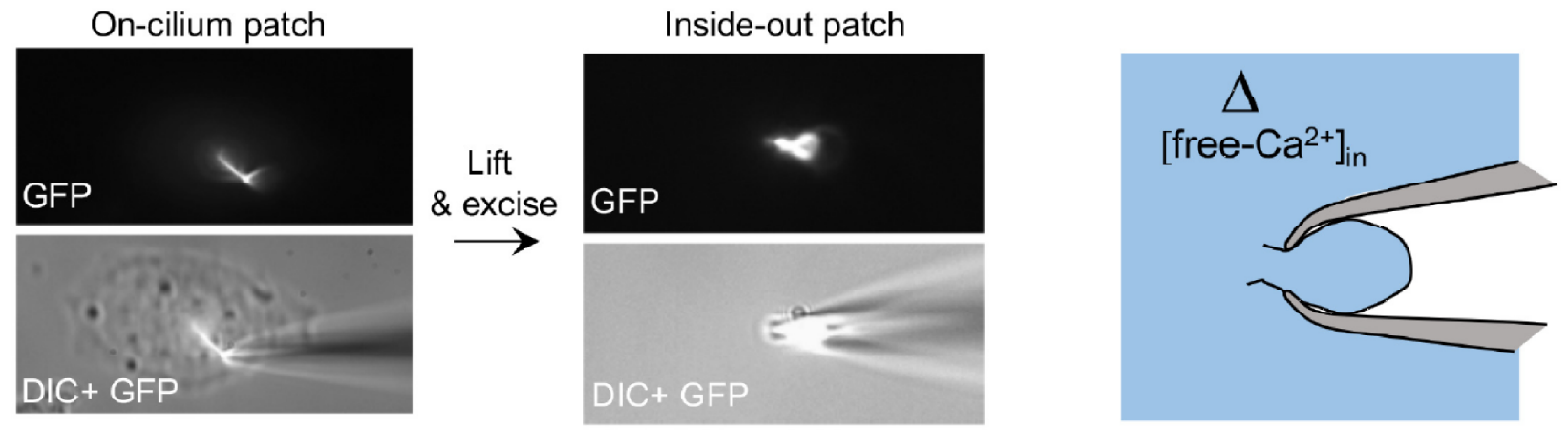

b

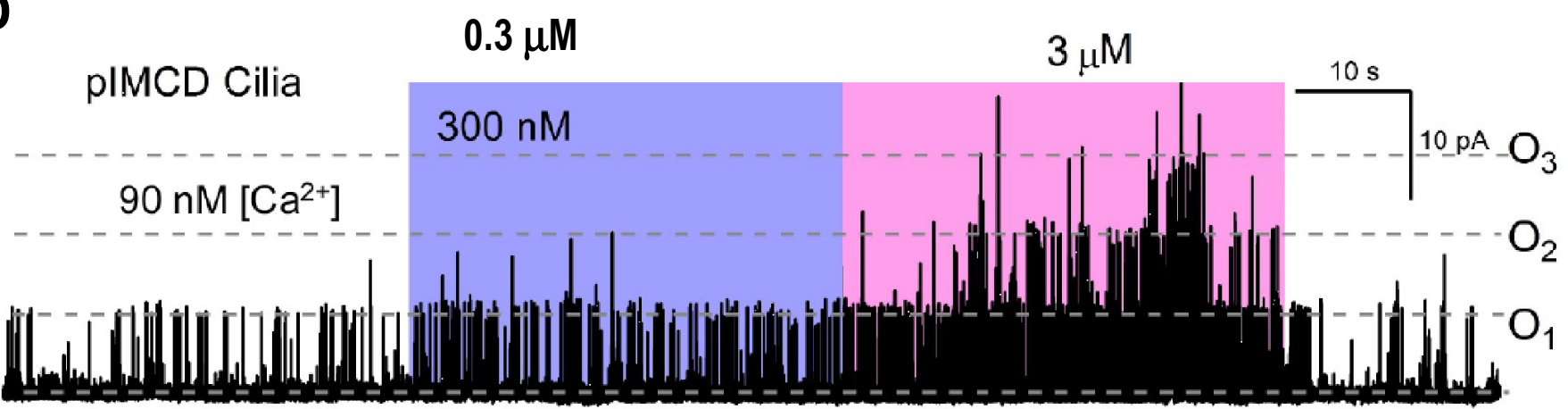

C

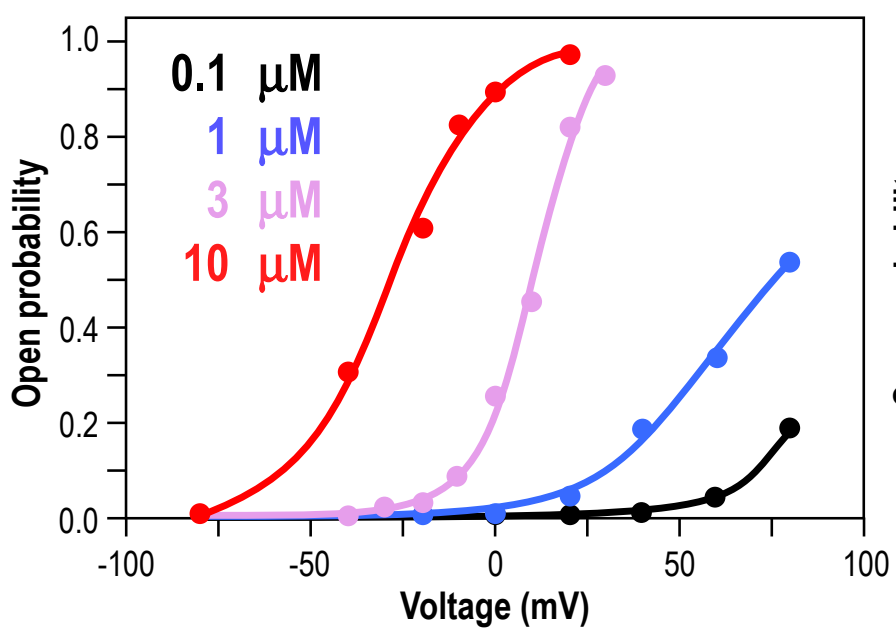

d

Endoplasmic reticulum

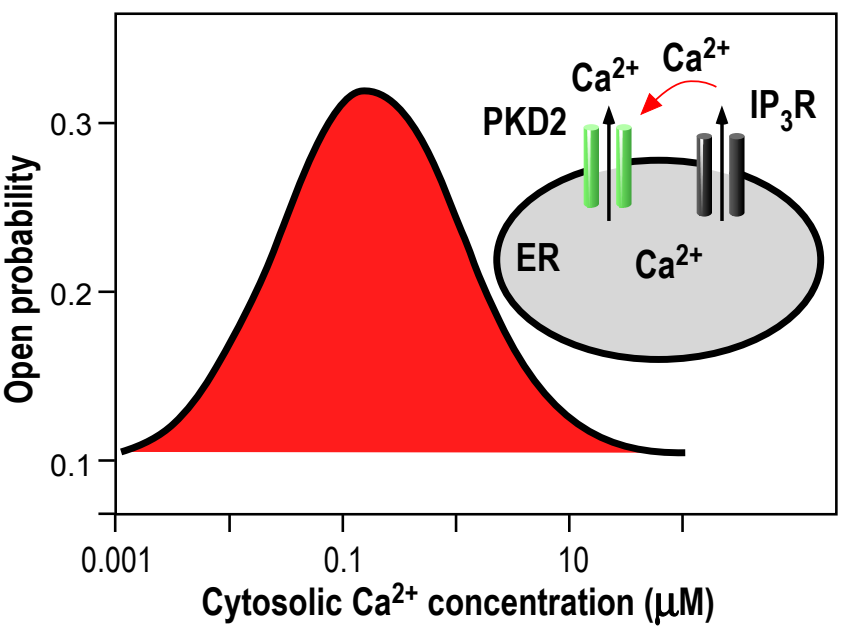

